\title{
Fast photometry at the Paris observatory: portable cameras and multi-fibre instruments
}

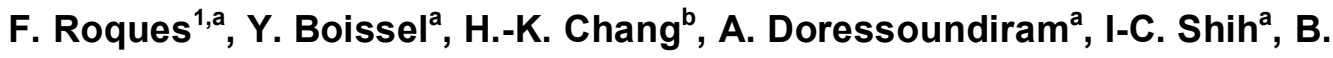 \\ Sicardy $^{\mathrm{a}}$, T. Widemann ${ }^{\mathrm{a}}$ \\ ${ }^{a}$ LESIA, Observatoire de Paris, 92195 Meudon, France \\ ${ }^{b}$ Institute of Astronomy, National Tsing Hua University, Hsinchu, Taiwan \\ E-mail: francoise.roquescobspm. fr
}

Occultations by distant objects of the Kuiper Belt can be done with two strategies

- To observe the occultations by known large objects which shadow path on Earth is predicted by astrometry

- $\quad$ To search for serendipitous occultations by smaller objects.

We have developped dedicated instrumentation for each strategy :

- a set of performant portable cameras for multi-sites observations of predicted occultations .

- multi-fibre instruments associated with rapid camera to perform fast accurate photometry on several targets in large field for serendipitous occultations. We have upgraded a 29 fibres spectrograph, Mefos, by replacing the spectrograph by a fast camera: Miosotys is mounted on the $1.93 \mathrm{~m}$ telescope of the Observatoire de Haute Provence. The second step is to build UltraPhot, a very performant instrument for multi-object fast photometry on the VLT, with between 60 and 150 fibres and a read-out frequency of $100 \mathrm{~Hz}$ in 3 colors. Scientific subjects of Miosotys and UltraPhot are numerous, serendipitous stellar occultations, but also young stellar objects, white dwarfs, exoplanet transits...

High Time Resolution Astrophysics IV - The Era of Extremely Large Telescopes-HTRA-IV Agios Nikolaos, Crete, Greece

May 5-7 2010

$1 \quad$ Speaker




\section{Introduction}

We propose to explore the largely unknown outer Solar System, and more precisely the trans-Neptunian objects (or Edgeworth-Kuiper Belt Objects, EKBO's) using the stellar occultation method. These objects are the most primitive in the Solar System, and they can help us understanding planetary formation processes.

One aspect of the project is the study of known EKBO's with predicted occultations. This would allow us to (a) monitor Pluto's and Triton's atmospheric seasonal variations over years, (b) measure sizes of several EKBO's at kilometric accuracy, and pin down density determinations, (c) discover other EKBO's with atmosphere. For this, we have built a set of fast cameras for multi-sites observations during occultation campaigns, and duplicate these cameras at low cost, to have them distributed in various institutes worldwide. We also require a $2 \mathrm{kx} 2 \mathrm{k}$ CCD camera for astrometric purposes, in order to improve our capability in predicting those events.

Another aspect of the project is the detection of serendipitous EKBO occultations, revealing 0.1 to $1 \mathrm{~km}$-sized objects during unpredicted events. This would allow us to (a) reconstruct the EKB (and possibly Oort cloud) structure from statistical studies of those occultations and (b) give the size distribution of those small bodies, an important parameter for constraining formation scenarii. For this, we want to develop fibre instruments on large telescopes, for simultaneous fast photometry of hundreds of stars.

\section{Beyond Neptune, an invisibile world}

Since the early 1990's [9], hundreds of new objects have been discovered beyond Neptune's orbit. Hypothesized in the 40's and 50's by K. Edgeworth and G. Kuiper, these Trans-Neptunian Objects (TNO's), or « Edgeworth-Kuiper Belt Objects », (EKBO's) are thought to be the remnants of the outermost population of bodies formed during the early solar system formation. Further perturbations by the giant planets have sculpted this population into various dynamical populations (classical disk, resonant «plutinos », scattered disk), see figure 1 and $[10 ; 11 ; 4,24]$.

One of the difficulties for studying those objects lies in their distances, more than $\sim 30$ Astronomical Units (AU). With diameter of $2500 \mathrm{~km}$ at most, those objects subtend angles smaller than $\sim 0.1$ arcsec on the sky, and usually much less, i.e. extremely small angular sizes when it comes to take any resolved images of their surfaces. Also, many of those objects are very faint (for instance, 10-km EKBO's have a magnitude of 30), requiring the largest telescopes (diameters $>8 \mathrm{~m}$ ) which have high schedule pressure. 


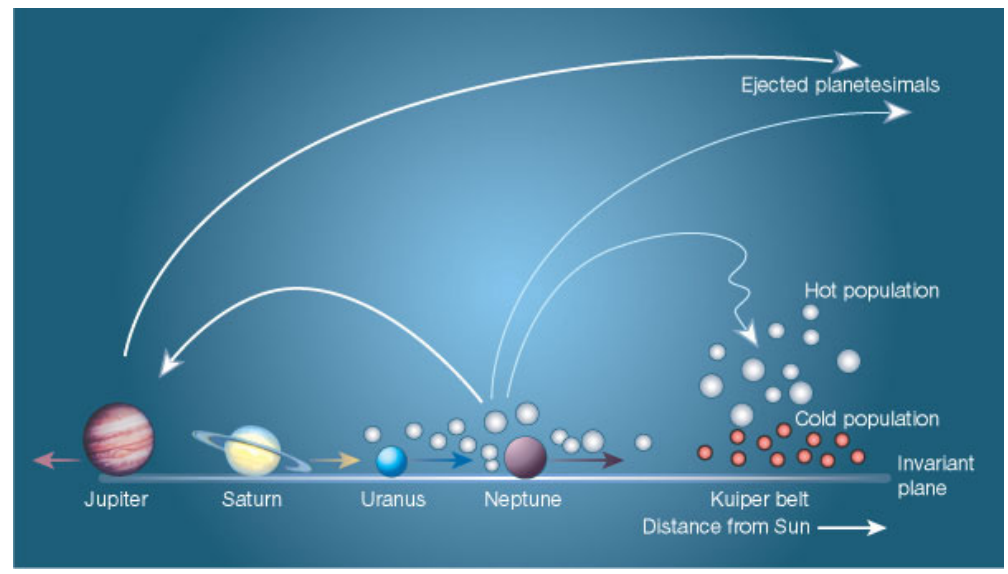

figure 1: Interactions of the giant planets with the primordial Edgeworth-Kuiper Belt (EKB). From Morbidelli and Levison [10].

Among various observational and theoretical tools designed to explore this remote region of the solar system are spectro-photometry studies to constrain surface composition, direct imaging with Adaptive Optics (AO) or space instruments (the Hubble Space Telescope or the Spitzer IR telescope), photometric light curves to derive rotational periods plus shapes, and theoretical dynamical studies aimed at evaluating the effects of the giant planets on the present EKB. Moreover, the NASA New Horizons Pluto Kuiper Belt mission will arrive at Pluto in July 2015, with a possible extended mission to another EKBO for a later date, but will focus its study on only two members of this very large population.

The stellar occultation method. Due to their motion on the sky, solar system objects may come in front of a star and occult it. Depending on size, this occultation may last from a small fraction of second to several hours. The star may then completely disappear if the body is opaque (e.g. a satellite), or may be partially attenuated by an atmosphere or semi-transparent object like a ring. Because angular diameters of stars are about one milli-arcsec (mas), their disk have typical sizes of a few $\mathrm{km}$ when projected at EKBO distances - typically 30-50 AU. This explains the success of stellar occultations in providing $\mathrm{km}$-scale resolution, out of reach of other methods. In some cases, if the projected stellar diameter is smaller than $1-\mathrm{km}$ (corresponding to fainter stars), then the resolution is eventually limited by the so-called Fresnel scale, $L_{F} \sim$ $\sqrt{ } \lambda D / 2$, where $\lambda$ is the observation wavelength and $D$ the distance of the EKBO, so that $L_{F} \sim 1 \mathrm{~km}$. 

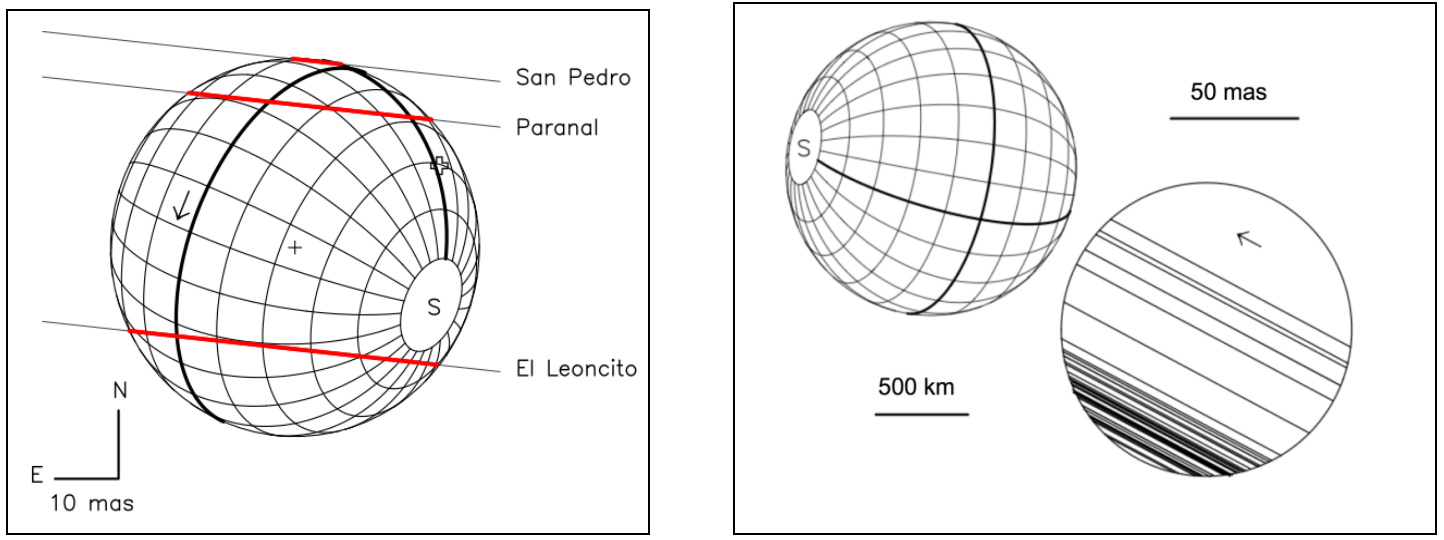

figure 2 : Left: occultation chords (red) obtained during the 11 July 2005 stellar occultation by Pluto's main satellite, Charon. The radius derived for Charon is $604 \pm 1.4 \mathrm{~km}$ [21]. Right: the 8 September 2001 occultation by Uranus' largest satellite, Titania, from which a radius of $788.3 \pm 0.65 \mathrm{~km}$ is derived [27]. Note the scales in milliarcsec (or " mas ").

Since early 1980's, our group has co-discovered Neptune's ring arcs in 1984 [6;19] and studied Uranus' narrow opaque rings. In 2001, we have put an upper limit for Titania's atmosphere [27]. In 2003, we have probed Titan's stratosphere a few months before the ESA/Huygens spacecraft's descent [22]. We revealed a drastic pressure variation in Pluto's atmosphere between 1988 and 2002 [20], accurately measured Charon's size in 2005 [21], and detect of the first serendipitous EKBO occultation [15].

We apply the stellar occultation method to both ends of the EKBO size spectrum. On one hand, we propose to observe stellar occultations by known EKBO's, for which ephemerides are available, so that stellar occultations are predictable. This involves bodies with diameters larger than $\sim 100-200 \mathrm{~km}$. On the other hand, we would like to detect very small EKBO's through serendipitous occultations, reaching subkilometric size objects.

Predictable occultations method are particularly efficient in providing sizes and shape at kilometric accuracy (figure 2). They can also reveal tenuous atmospheres around EKBO's, at levels of 20 nbars or less. We want in particular to monitor Pluto's and Triton's varying atmospheres and discover atmospheres around other EKBO's. Accuracy and sensitivity of this method are out of reach of any other methods (except of course space missions). Serendipitous occultations, on the other hand, have no other competing methods, as the magnitudes of the corresponding objects, $R \sim 35$ or fainter, is unreachable through classical ground-based imaging. Such occultations reveals the vertical and radial distribution of the EKBO's as far as $50 \mathrm{AU}$ and beyond. Also, it provides information on size distribution down to $100 \mathrm{~m}$ in size, a key parameter for better understanding formation processes in this remote region of the solar system (figure 8). 


\section{Two objectives}

They both concern the EKBO's, but at the two extremities of their size distribution (see figure 3).
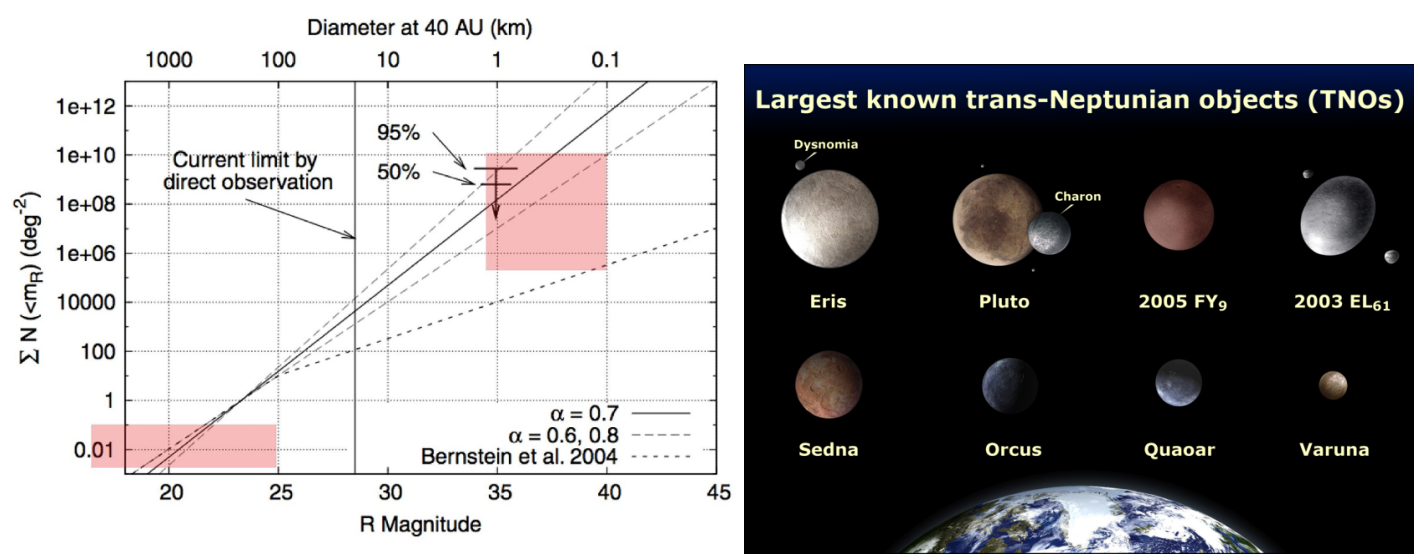

figure 3 : Left: Various possible size distributions in the EKB. Figure taken from [2]. The overlaid red regions delineate the typical size intervals that we want to explore in our project. Right: typical sizes of a few large EKBO's. Note that some of those bodies have satellites.

The first objective is to observe stellar occultations by the largest EKBO's. This includes Eris, Pluto, Quaoar, etc..., and also Neptune's main satellite, Triton. The latter is probably a captured body, which is akin to Pluto both in size and atmospheric composition. Scientific goals pursued are :

(a) Get sizes and shapes at kilometric accuracy, as we already did for Charon and Titania. Current published sizes are based on visual magnitudes combined with IR thermal emession, and have accuracies of $\sim 5-20 \%$ (i.e. several tens of $\mathrm{km}$ ), a value that we could pin down dramatically. For those bodies with satellites, constraints on masses are available from orbital motion. Thus, accurate sizes translate into accurate densities, an important parameter to assess internal structure models (like ice/rock ratio).

(b) Discover and/or monitor atmospheres around the largest EKBO's. For instance, Pluto's atmosphere has suffered a dramatic surge of pressure by factor of two between 1988 and 2002 ([20] and figure 7). Overall, two orders of magnitude differences in pressure are expected between Pluto's perihelion and aphelion, revealing a complex interplay between ices sublimation, changing solar insolation as the object revolves around the Sun, and albedo variations.

(c) Scan the surroundings of these objects, in search of satellites. Probability are small, but such detections come «for free » when a campaign is organized for a given occultation. Again, kilometric accuracies would be guaranteed. 
The technique comes in support/complements space-borne in-orbit instrumentation or mission (e.g. Cassini/VIMS stellar occultations, Huygens/HASI) or deep solar system fly-by missions (New Horizons, which will fly by the Pluto-Charon system in July 2015), as well as space-based observatories (Spitzer, HST). Recent discovery of Pluto's two small moons, Nix and Hydra with HST [26] suggest the possibility that the Pluto system could possess tenuous rings created from Kuiper-belt debris impacts onto the surfaces of these satellites. Therefore ground-based stellar occultation measurements have increased significance in the context of New Horizons fly-by in 2015.

This project, however, has its own challenges. One of them is being able to predict occultations by large EKBO's, knowing that their typical angular diameter on the sky is 30-100 milli-arcsec (mas), and their distance is 30-50 AU's. At such distances, 1 arcsec subtends $\sim 20,000-30,000 \mathrm{~km}$. So, an error of 0.1-0.3 arcsec in the body ephemeris (typical of EKBO's) is enough to displace the occultation shadow track by thousands of $\mathrm{km}$. This is a large uncertainty when it comes to plan observations months in advance of both large, fixed, and small, portable telescopes.

The second objective is to detect serendipitous occultations by small (i.e. sub-km in size) EKBO's, by monitoring at high rate (typically 40-100 data point/sec) hundreds of stars simultaneously for several hours. Scientific goals are now :

(a) Explore the lower end of size distribution in the EKB, in the range 0.1-1.0 km. Various models (figure 8 and $[1 ; 7]$ ) predict various size distributions. The important point is that several events are expected per night, by monitoring hundreds of properly chosen stars. The statistics can thus be rich enough to discriminate between various models.

(b) use stellar fields of view (FOV) at various ecliptic latitudes to recover the vertical and radial distributions of EKBO's around the invariable plane, e.g. measure the vertical extension of the " cold» population (figure 1), estimate the population in the « hot» population, etc... By the same token, changing the orientation of the FOV with respect to Earth motion may be a powerful way to test the reality of the detected bodies, i.e. their actual belonging to the EKB. Note that because diffracting patterns are distance-dependent, serendipitous occultations allow us to estimate the distance of the detected EKBO's (figure 8 and [16]). A challenging by-product of our method would even be the first detection ever of a $\sim 3 \mathrm{~km}$ Oort cloud object at 10,000 AU, a feasible observation using serendipitous occultations.

This information is critical for better understanding the planet formation stage. In particular, the primordial structure of the proto-planetary disk just outside the giant planet region has been deeply modified by planetary migrations, resonance trapping and collisions. A better knowledge of the Kuiper Belt will allow comparisons with circumstellar material around other stars, and provides some clues on the comet belt observed around the $\beta$-Pictoris system, and rings or disk-like features akin to Kuiper belts around other young stars. 
The main challenges of serendipitous occultations by small EKBO's are the high rate of acquisition (typically 40-100 data points/sec) and large FOV's to follow hundrerds of stars at the same time. This precludes large 2-D arrays, which end up in untractable storage problems and mass analysis. The available fast cameras that we used (e.g. Ultracam) rapidly reached their limits due to small FOV, and therefore, too small numbers of occultation detections for proper statistical analysis. Fibre instruments give simultaneous aperture photometry of hundreds stars, recorded as fast as $100 \mathrm{~Hz}$. Those instruments allow long-term dedicated surveys.

\section{Predicted occultations}

In order to maximize our success rate when observing stellar occultations by known EKBO's, we have to pin down the astrometric accuracy on both the star and the occulting body down to 20-30 mas. We have long term collaborations with a team at Geneva Observatory, Switzerland (R. Behrend et al.) and a team at Rio de Janeiro Observatory, Brazil (M. Assafin, R. Vieira Martins et al.). These teams use 1.2 to $2.2 \mathrm{~m}$ class telescopes in Chile (La Silla) and Brazil (Pico dos Dias), measuring star positions against UCAC2 star catalog in contiguous FOV's covering the regions crossed by the occulting body, (see figure 4). By the same token, offsets with respect to ephemeris for those bodies are evaluated, and projected into the future. This is crucial, as ephemeris errors of, say, 100 mas result in displacements of thousands of $\mathrm{km}$ on the ground.

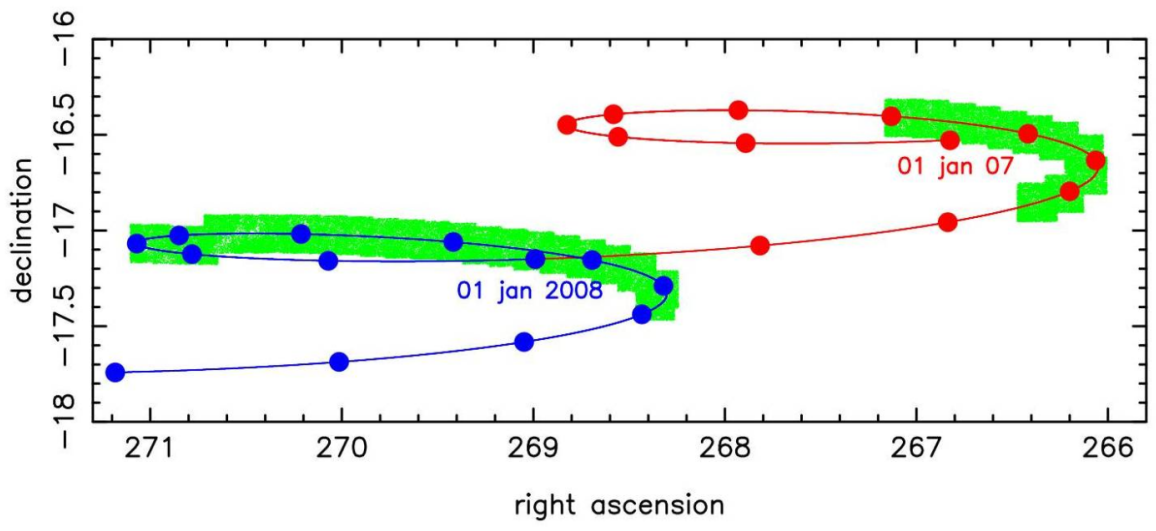

figure 4 : Prediction star catalog by R. Behrend (Geneva Obs.), using the $1.2 \mathrm{~m}$ "Euler" Telescope at La Silla (Chile). More than 180,000 star positions have been measured along Pluto's path in 2007 and 2008, with an absolute accuracy of 30-40 mas, using the UCAC2 catalog. Each red or blue dot corresponds to the beginning of a month. Once specific candidates are found, more accurate measurements are made, at the 20 mas level. Similar work is made by a Brazilian team (Rio de Janeiro Obs.). Various bodies are involved (Pluto, Triton, Eris, Quaoar, Orcus, Varuna, etc...) 


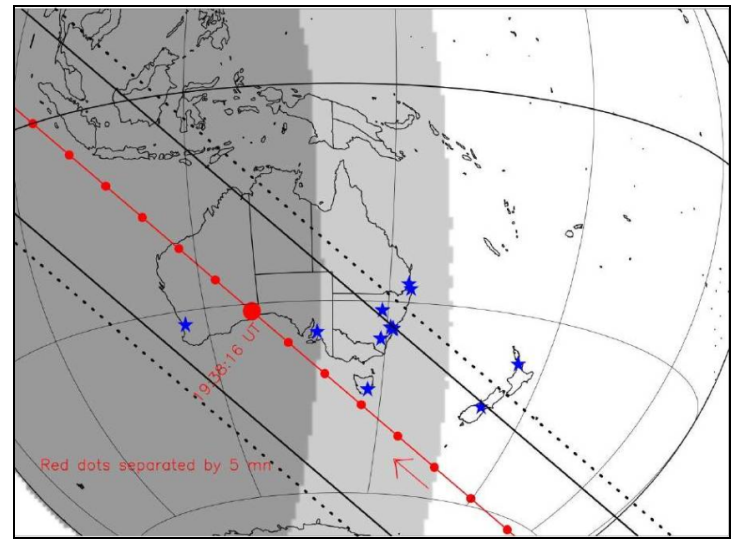

figure 5 : Left: prediction of path of Pluto's shadow on Earth on 5 April 2008. Solid black lines are the projections of Pluto's geometric shadow, dotted lines show the extension of Pluto's atmosphere, and red dots mark 5-mn time interval along central line. Blue star symbols are expected stations where we will get observations.

Our following step is to organize campaigns to observe the events for various sites, using both large (e.g. VLT) and small (1-m telescopes, or even amateur instruments down to $0.3 \mathrm{~m}$ ). figure 5 shows examples of such campaigns involving Pluto in 2008.

On 2010, February 19, Varuna occulted 2UCAC star 41014042, as seen from regions in southern Africa and north-eastern Brazil. Among the observations, three were negative, and two positive, one from Camalau (visual), and one from São Luis (CCD). This makes Varuna the most remote solar system object observed to date through a stellar occultation, with a geocentric distance of 6.4 billions $\mathrm{km}$. The São Luis occultation has a duration of $52.5+/-0.5 \mathrm{sec}$, corresponding to a chord length of $1003+/-9 \mathrm{~km}$ projected in the plane of the sky. Combining the positive and negative observations, we may already conclude that Varuna is either spherical and much bigger that expected (i.e. with a diameter of about 1000-1100 km), or, more probably, highly elongated. Furthermore, no atmospheric signature is apparent in the light curve [23].

As an example of potential scientifc return on EKBO's, we consider a Pluto occultation light curve obtained in 2002 (see figure 6). The observed flux variations are due to differential refraction of stellar rays in tenuous Pluto's nitrogen $\left(N_{2}\right)$ atmosphere. The start of occultation corresponds to typical pressure levels of $<\sim 0.1 \mu$ bar, while the bottom - at mid-event - correspond to pressures of $\sim 10 \mu$ bar. Beyond a smooth variation, there are fast signal fluctuations, or "spikes », caused by local density inhomogeneities. figure 7 also shows the pressure profile derived from this light curve. It is extracted through an Abel inversion method, where an " onion layer » iterative procedure uses geometric optics refraction equations, plus ideal gas and hydrostatic equations to provide $T(r)$, the temperature as a function of distance to planet's center.

An interesting feature of $T(r)$ is the roughly isothermal structure at high altitude, followed by a sudden cooling as the surface is approached. This strong inversion layer is due to conduction, as the cold surface is at $\sim 40 \mathrm{~K}$, due to solar insolation, while 
higher levels are heated up to $\sim 100 \mathrm{~K}$ by IR absorption by methane $\left(\mathrm{CH}_{4}\right)$, a minor species in Pluto's atmosphere (see e.g. [24]). Also shown is the profile of temperature gradient, $d T / d r$ as a function of $r$. Note the local fluctuations causing the spikes in the light curve. They reveal a dynamical activity, either due to shear instabilities caused by strong winds, or internal gravity waves associated with orographic activity.

A remarkable result obtained during that observation is that pressure increased by a factor of two between 1988 and 2002, see figure 7. This is paradoxical as Pluto has been receding from Sun, thus cooling, and the $N_{2}$ atmosphere is thought to be at vaporpressure equilibrium with the cold $(\sim 40 \mathrm{~K}) N_{2}$ ice at the surface. The vapor-pressure is so sensitive to temperature, that a temperature decrease of $0.6 \mathrm{~K}$, expected from Pluto's recession between 1988 and 2002, should result in a pressure decrease by a factor of 1.4 , while an increase by a factor of $\sim 2$ is actually observed.

This means that more complex scenarii are at work, where seasonal effects associated with the recent passage through equinox may lead to redistribution of ices on Pluto's surface. For instance, the recently sunlit southern cap could now sublimate its nitrogen ice, thus feeding the atmosphere with more $N_{2}$ in spite of the decreasing solar energy available. A time lag is now necessary for this nitrogen
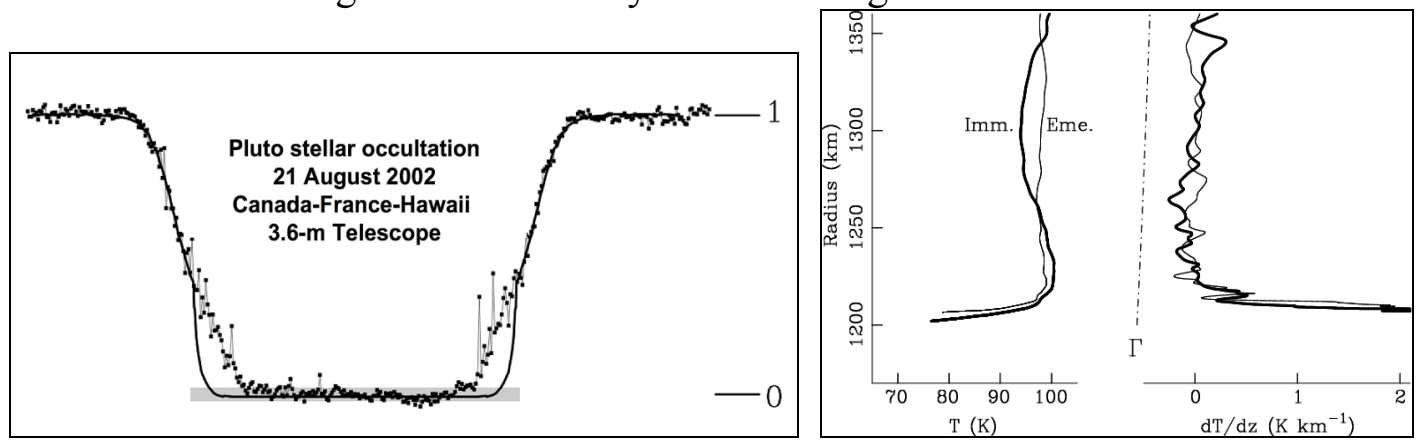

figure 6 : Left: stellar light curve obtained during the 21 August 2002 stellar occultation by Pluto. The flux is normalized between 1 (full stellar flux) and 0 (star completely gone). Total duration is about $7 \mathrm{mn}$. The continuous solid line is the expected light curve if Pluto's atmosphere had been unchanged since 1998.. Right: Temperature profile, $T(r)$, and temperature gradient $d T / d r$ derived from the ligth curve shown at left (from [20]).

to condense near the now permanently unilluminated northern polar region, as anticipated by Hansen and Paige [5], see figure 7. Note, however, that Hansen and Paige overestimate the 1988-2002 increase by a factor of two. This stems from high sensitivity of models to some poorly known parameters, like thermal inertia, albedo features distribution, degree of mixtures if various ices, etc...

This is why it is now important to monitor Pluto's atmospheric activity to discriminate between various models. It is also important to follow up that activity as the NASA New Horizons Pluto Kuiper Belt mission approaches the planet, with a fly-by expected in July 2015. 

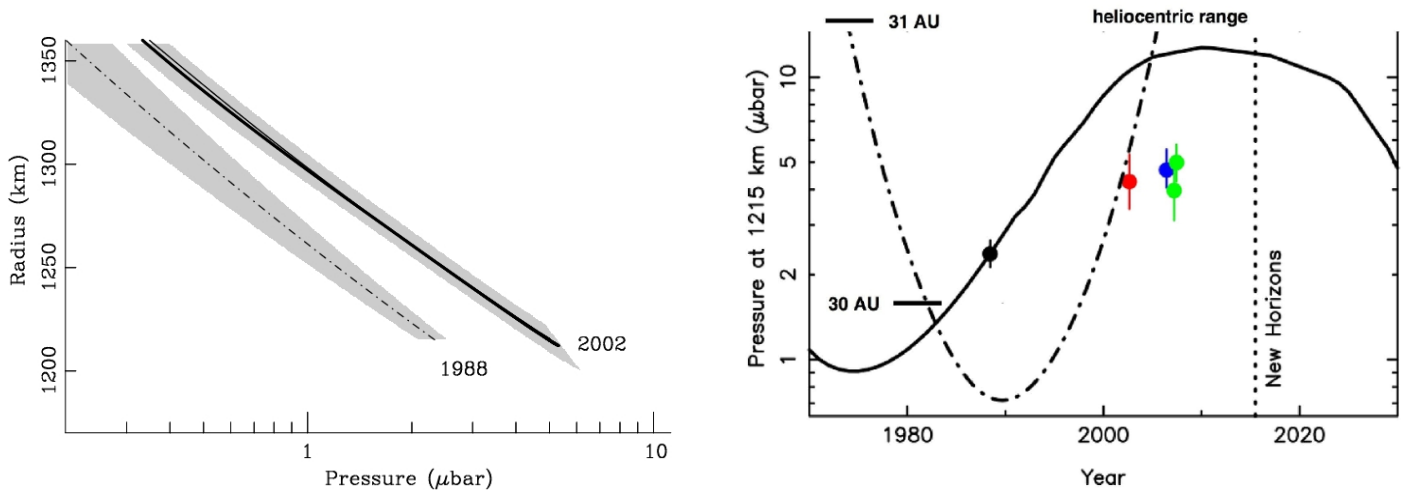

figure 7 : Left: Pluto's pressure profile - in logarithmic scale - obtained in 2002 (from [20]), and 1988 [28]. Grey areas are uncertainty domains. Note the doubling of pressure observed in 14 years. Right: pressure at $1215 \mathrm{~km}$ from Pluto's center as a function of time. Colored points with error bars are pressure measured by our team during 2002-2007, compared with the previous measure of 1988 (black dot, from [28]). The solid line the theoretical prediction by Hansen and Paige. Dash-dotted line is Pluto's heliocentric distance. It shows that although Pluto has been receding from Sun since 1989, and thus cooling, complex seasonal effects have increased the pressure by a factor of about two between 1988 and 2002.

Other EKBO's have sizes comparable to a larger than Pluto, figure 3. We would like, for the first time ever, measure their size at kilometric accuracy. The ray tracing models we developed can now be used for detection of plumes or possible season-induced tenuous atmospheres around specific EKBO's, which should have retained substantial amount of their initial volatile inventory. Quaoar and 2005 FY9, in particular, have sizes intermediate between Pluto's and Charon's (figure 3). Although not measured on Quaoar, $\mathrm{CH}_{4}$ ice has been observed on $2005 \mathrm{FY}$, whose distance to Sun varies from 53 to 38.5 UA. Future occultations by distant EKBO's will allow to put an upper limit on any sublimation or cryogenic gaseous material.

Parallel to those campaigns, we maintain complementary ongoing programs on Pluto. For instance, Run 381.C-0247 with VLT/CRIRES in 2008 (Lellouch et al.) could detect gaseous $\mathrm{CH}_{4}$ in Pluto's atmosphere. Constraints on temperature near Pluto's surface could be gained, which would confirm or reject the hypothesis of a troposphere near the surface. A troposphere would imply a «small» radius (e.g. $1140 \mathrm{~km}$ ) for Pluto, and therefore a «large » pressure (e.g. $40 \mu \mathrm{bar}$ ) at the surface. An absence of troposphere would in contrast favor « large » radii (say $\sim 1175 \mathrm{~km}$ ), and surface pressure around 10 $\mu$ bar. Also, given the variability of Pluto's atmosphere, these data will provide a reference point for the $\mathrm{CH}_{4}$ measurements during the New Horizons flyby in 2015. 


\section{Serendipitous occultations}

Very small objects of the Kuiper Belt are invisible but can be detectd by serendipitous occultations (figure 8 . We did theoretical analysis $[12 ; 13]$, and made observations with a multi-photometer instrument at the Pic du Midi «Bernard Lyot» $2 \mathrm{~m}$ telescope [14], and with Ultracam at the $4.2 \mathrm{~m} \mathrm{WHT} \mathrm{(La} \mathrm{Palma)} \mathrm{and} 8.2 \mathrm{~m} \mathrm{VLT} \mathrm{(Paranal).} \mathrm{Some}$ occultation events have been detected with Ultracam [15], in RXTE observations [8] and in HST archival data [17]
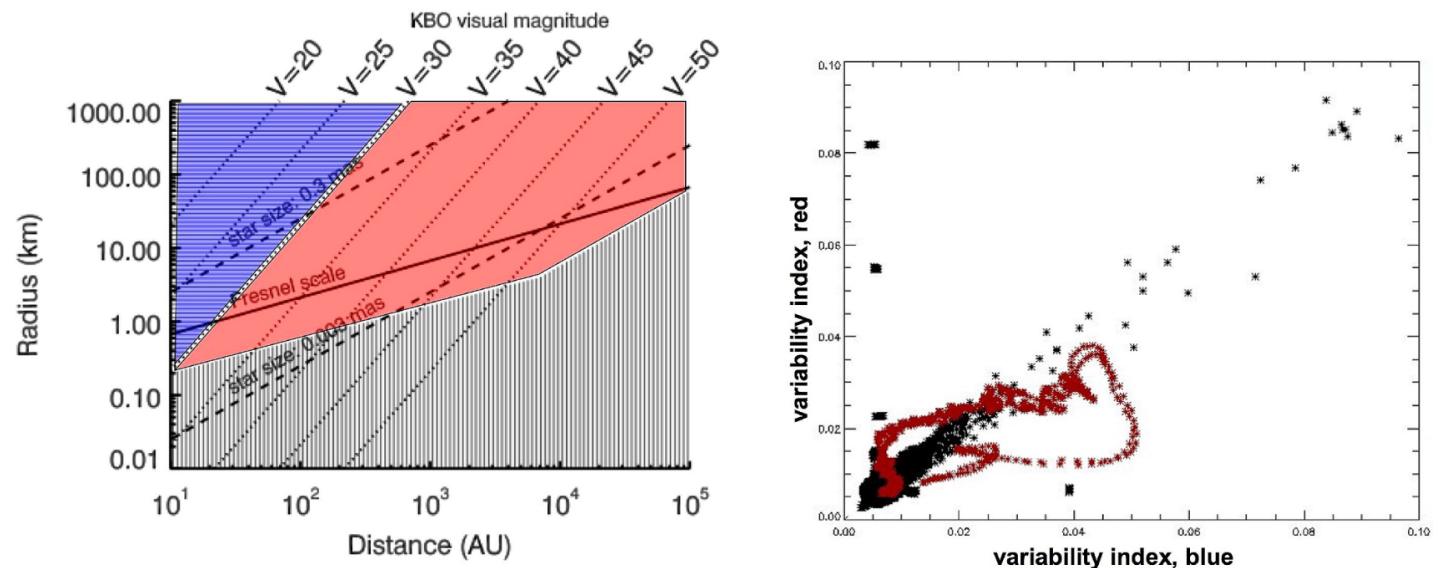

figure $8:$ Left: the red area is the zone accessible by the serendipitous occultation method in a distance-size diagram. The blue zone is the zone accessible by direct imaging. Serendipitous occultations can thus detect smaller objects and farther away than direct imaging. The lower grey zone is unaccessible to any current methods [16]. Right: analysis of rms fluctuations in simultaneous light curves taken in two colors, during one night of $V L T$ with the Ultracam camera (" variability indexes "). It reveals several kinds of events. Some of them, in black, are detected in only one channel (e.g. electronic problems), diagonal events are correlated clouds or seeing deterioration. Red points correspond to the synthetic signature of a 200-m object at $50 \mathrm{AU}$ [16].

The multi-fibres photometry concept. The association of fast photometry with a multi-fibres instrument and a large telescope provides a unique possibility to perform fast accurate photometry on several targets in large fields.

An australian team [3] used the fibres spectrograph 6df in «straight thru » mode on the UKST (Siding Spring). However, the small aperture of the $6 \mathrm{df}$ fibres on the sky induces noise difficult to discriminate from real occultation events because there is no spatial, nor spectral information .

Our team has a plan in two steps :The first step is to upgrade a 29 fibres spectrograph formely at the ESO 3.6-m telescope, by replacing the spectrograph by a fast camera. The instrument, Miosotys is mounted on the $1.93 \mathrm{~m}$ telescope of the Observatoire de Haute Provence [18]. The second step is to build a dedicated instrument, UltraPhot, for the UT2 « Kueyen » Very Large Telescope at ESO. UltraPhot will provide, at short term 
( $\sim 2$ years) a very performant instrument for multi-object fast photometry in the southern hemisphere. UltraPhot would use the fibre positioning system of the UT2, and would have access to the entire field of the Nasmyth focus ( 25 arcmin diameter). The number of fibres of UltraPhot will be between 60 and 150 for a read-out frequency of $100 \mathrm{~Hz}$ in 3 colors. The expected $\mathrm{S} / \mathrm{N}$ ratio could be at least $\sim 10^{3} / \sqrt{ } f$, where $f$ is the read-out frequency for a magnitude 13 star.

\section{Conclusions}

Stellar occultations is a very powerful method for exploring the invisibile outer solar system. The larger bodies are studied with predicted occultations and the population of smaller bodies are explored with serendipitous occultations. The scientific results are numerousous:

- monitor Pluto's and Triton's atmospheric changes, and refine under-constrained models of seasonal evolution for those bodies. This in turns yields information on frost distribution and albedo features, allowing more accurate predictions for future pressure changes and refine our general understanding of surface/atmosphere equilibrium for other similar bodies ;

- measure sizes of other EKBO's at kilometric accuracy. Combined with masses given by satellite motions, this yields accurate density values, from which we constrain ice/rock ratio and therefore, physical conditions prevailing during the formation of EKBO's.

- discover one or several EKBO's with sublimating surfaces, and compare them with Pluto's and Triton's atmospheres. Constrain ice surface composition and sublimation models.

- get EKBO's serendipitous occultations on a survey basis, to reconstruct the EKB, until the Oort cloud. Derive radial and vertical structure from statistical studies of those occultations.

- obtain the size distribution of $1 \mathrm{~km}$-sized or smaller EKBO's, from which important constraints on collisional history and formation scenarii for the outer Solar System can be obtained.

Two types of fast photometry instruments are used depending on the approach:

(a) set of portable accurate cameras with reliable time reference for multi-sites observations of predicted occultation,

(b) fibres instruments with rapid cameras on large field, with some spatial definition and/or spectral information, necessary to filter noise in the ligthcurves. Such instrument $\mathrm{s}$ have several scientific returns in others domains than solar system study, as exoplanets transits, $\mathrm{X}$ binaries, young stellar objects, etc....

This work is supported by the grant ANR BLAN08-2_328288 and NSC 98-2923-M007-002-MY3 under the project "Beyond Neptune". Facilities : ESO-VLT, OHPT193 


\section{References}

[1] Bernstein et al., Astron. J., 128, 1364, 2004

[2] Bickerton, Kavelaars \& Welch, Astron. J. 135, 1039, 2008

[3] Georgevits, Bull. Am. Astron. Soc., 38, 37.072006

[4] Gomes, Nature 426, 393, 2003

[5] Hansen and Paige, Icarus 120, 247, 1996

[6] Hubbard et al., Nature 319, 636, 1986

[7] Kenyon and Bromley, Astron. J. 128, 1916, 2004

[8] Liu et al., Mon. Not. R. Astron. Soc. 388, L44-L48 2008

[9] Luu and Jewitt, Nature 362, 730, 1993

[10] Morbidelli and Levison, Nature 422, 30, 2003

[11] Morbidelli and Levison, Nature 426, 419, 2003

[12] Roques et al., Astron.J.93, 1549, 1987

[13] Roques and Moncuquet, Icarus 147, 530, 2000

[14] Roques et al., Astrophys. J. 594, L63, 2003

[15] Roques et al., Astron. J. 132, 819, 2006

[16] Roques et al., in The Solar System beyond Neptune (eds Barucci et al.), UoA Press, 2008

[17] Schlinting et al., Nature, 462, 2009

[18] Shieh et al, in proceedings of SPIE conference, 2010

[19] Sicardy et al., Icarus 89, 220, 1991

[20] Sicardy et al., Nature 424, 168, 2003

[21] Sicardy et al., Nature 439, 52, 2006

[22] Sicardy et al., J. Geophys. Res., 111, E11S91, 2006

[23] Sicardy et al. $B A A S$, in press, 2010

[24] Strobel and Zhu, Icarus 120, 266, 1996

[25] Tsiganis et al., Nature 435, 459, 2005

[26] Weaver et al., Nature 439, 943, 2006

[27] Widemann et al., Icarus, 199, 458, 2009

[28] Yelle and Elliot, in Pluto and Charon (eds Stern and Tholen), 347, UoA Press, 1997 